\title{
SYMPOSIUM 2: LANGUAGE AND NUMERICAL SKILLS
}

\author{
Organized by Luisa Girelli and Carlo Semenza \\ Department of Psychology, University of Trieste, Italy
}

The aim of this symposium is to examine the relationship between language and numerical skills by providing an overview of the most recent functional imaging and neuropsychological studies. The recent development of neuroimaging methods indeed provides a powerful tool for investigating the interplay of these two cognitive functions. A further source of information comes from the new interest in the neuropsychology of disorders such as the Turner's syndrome. This condition provides, in fact, the opportunity to study a genetically determined dissociation between linguistic and mathematical skills.

In the introductory presentation, Delazer and Girelli will outline the historical background and current issues regarding the relationship between language and numerical processing. Brian Butterworth and co-workers will present data on the linguistic and numerical abilities of subjects with Turner's syndrome: this genetic abnormality is known to be associated with poor mathematical performance. The observed cognitive profiles will be discussed in light of both neuroanatomical and genetic data. Thiuox, Seron, and Pesenti have examined, using PET, the neuroanatomical substrates of numerical semantic processing and they have directly confronted processing of number and processing of words in the living category. Code and collaborators will report the outcome of their PET study on the cerebral circuits supporting mental calculation; their attention is paid to separate linguistic and nonlinguistic components. Finally, Dehaene and Cohen will provide fMRI data elucidating the cerebral networks engaged in various numerical tasks. In particular they distinguish and localize language-specific and quantity-specific representations mediating simple arithmetic.

Outline of the symposium (Chair: Carlo Semenza).

1. Margarete Delazer and Luisa Girelli. Language and numerical skills: An introduction to past and present issues.

2. Brian Butterworth, Alessia Granà, Manuela Piazza, Luisa Girelli, Cathy Price, and David Skuse. Language and the origins of number skills: Karyotypic differences in Turner's syndrome. 
3. Marc Thiuox, Xavier Seron and Mauro Pesenti. Functional neuroanatomy of the semantic system: The case of numerals.

4. Chris Code, Simon Cowell, Egan Gary, John Watson, and Jenny Harasty. Mental Calculation and number word repetition: A PET study.

5. Stanislas Dehaene and Laurent Cohen. Language and elementary arithmetics: Dissociations between operations.

Proposed time allotment (the organizers are ready to accommodate the time allotment according to the Committee suggestions). Time allotment will be $20 \mathrm{~min}$ for each speaker and $10 \mathrm{~min}$ for the introductory talk (Delazer and Girelli). Each paper will be followed by a 10-min discussion.

\section{Language and Numerical Skills: An Introduction to Past and Present Issues}

\section{Margarete Delazer* and Luisa Girelli*, ${ }^{*}$}

*University Klinik fuer Neurologie, Innsbruck, Austria; and †Department of Psychology, University of Trieste, Italy

The issue of the relationship between language and numerical skills has a long tradition in neuropsychology; indeed, the very first descriptions of disorder in calculation date from the last decades of the 19th century, thanks to researchers studying aphasia (e.g., Bastian, 1898). They noticed that aphasic patients occasionally presented the inability to read and write numbers and to calculate, and these difficulties were considered part of the aphasic syndrome. Yet, since these early contributions the dissociation between language and numerical processing has not been neglected. Berger (1926) distinguished between primary and secondary acalculia; the latter referred to calculation problems associated with aphasia. Similarly, Hecaen and coworkers (1961) distinguished calculation problems secondary to alexia or agraphia from pure anarithmetia. Thereafter, the clinical investigation of numerical disorders has gradually acquired autonomy from the study of linguistic functions, thanks to the more and more frequent observations of impaired calculation abilities in absence of any language deficits (Warrington, 1982) and vice versa (Hermelin \& O'Connor, 1990). In recent years, the growing numbers of systematic studies focused on deficits in number processing and/ or calculation have provided evidence for, on one hand, establishing the functional autonomy of numerical abilities from other cognitive functions and, on the other hand, identifying the multiple cognitive mechanisms underlying these competencies. All current models draw a major distinction between the number processing and the calculation systems. Moreover, they all postulate functionally independent mechanisms mediating numeral comprehension and numeral production as well as distinct pathways for processing Arabic 
and verbal numerals (e.g., McCloskey, 1992; Cipolotti \& Butterworth, 1995; Dehaene, 1992).

It is, indeed, thanks to our improved understanding of the cognitive mechanisms mediating number processing and calculation abilities that we can now look to the interplay of numerical and linguistic functions with new insight. In fact, though double dissociations between language and numerical abilities have been reported (e.g., Rossor, Warrington, \& Cipolotti, 1995; Hittmair-Delazer, Seiler, \& Benke, 1995), it is indisputable that some numerical abilities are mediated or preferentially supported by linguistic functions (e.g., counting, reading numbers). Moreover, numerical skills are mediated by different notational systems for representing quantities which are, just as language, regulated by lexical, semantic, and syntactic mechanisms. However, it remains to clarify the extent to which processing and representations of the numerals differ from processing and representations of other linguistic (and semantic) categories.

The aim of this symposium is to examine the relationship between language and numerical skills by providing an overview of the most recent functional imaging and neuropsychological studies. The recent development of neuroimaging methods indeed provides a powerful tool for investigating the interplay of these two cognitive functions. A further source of information comes from the new interest in the neuropsychology of disorders such as the Turner's syndrome. This condition provides, in fact, the opportunity to study a genetically determined dissociation between linguistic and mathematical skills.

Brain Butterworth and co-workers have extensively investigated linguistic and numerical abilities in subjects with Turner's syndrome: this genetic abnormality is known to be associated with poor mathematical performance. The observed cognitive profiles are discussed in light of both neuroanatomical and genetic data.

Thiuox, Seron, and Pesenti have examined, using PET, the neuroanatomical substrates of numerical semantic processing and directly confronted processing of number and processing of words in the living category.

Code and collaborators investigated, in a PET study, the cerebral circuits of mental calculation by separating linguistic and non linguistic components.

Finally, Dehaene and Cohen will provide fMRI data elucidating the cerebral networks engaged in various numerical tasks. In particular they distinguish and localize language-specific and quantity-specific representations mediating simple arithmetic.

\section{REFERENCES}

Bastian, H. C. 1898. A treatise on aphasia and other speech defects. London: Lewis

Berger, H. 1926. Über Rechenstörungen bei Herderkrankungen des Großhirns. Archiv für Psychiatrie und Nervenkrankheiten, 78, 238-263. 
Cipolotti, L., \& Butterworth, B. 1995. Toward a multiroute model of number processing: Impaired transcoding with preserved calculation skills. Journal of Experimental Psychology: General, 124, 375-390.

Dehaene, S. 1992. Varieties of numerical abilities. Cognition, 44, 1-42.

Hecaen, H., Angelergues, T., \& Houiller, S. 1961. Les varietes cliniques des acalculies au cours des lesions retroronlandiques. Revue de Neurologie, 105, 85-103.

Hermelin, B., \& O' Connor, N. 1990. Factors and primes: A specific numerical ability. Psychological Medicine, 20, 163-169.

Hittmair-Delazer, M., Seiler, U., \& Benke, T. 1995. Impaired arithmetical facts but intact conceptual knowledge: A single-case study of dyscalculia. Cortex, 31, 140-147.

McCloskey, M. 1992. Cognitive mechanisms in numerical processing: Evidence from acquired dyscalculia. Cognition, 44, 107-157.

Rossor, M. N., Warrington, E. K., \& Cipolotti, L. 1995. The isolation of calculation skills. Journal of Neurology, 242, 78-81.

Warrington, E. K. 1982. The fractionation of arithmetical skills: A single case study. Quarterly Journal of Experimental Psychology, 34A, 31-51.

\section{Language and the Origins of Number Skills: Karyotypic Differences in Turner's Syndrome}

\section{Brian Butterworth,* Alessia Granà*, $\uparrow$ Manuela Piazza,* Luisa Girelli*, ${ }^{*}$ Cathy Price, $\neq$ and David Skuse $\S$}

*Institute of Cognitive Neuroscience, $\$$ Wellcome Department of Cognitive Neurology, and §Institute of Child Health, University College London, United Kingdom; and †Department of Psychology, University of Trieste, Italy

Evidence from studies of neurological patients suggests a double dissociation between language ability and numerical performance. Cipolotti, Butterworth, and Denes (1991) report a stroke patient with excellent language but a very dense acalculia. Conversely, Rossor, Warrington, and Cipolotti (1995) describe a patient with Pick's disease who can manage only the most rudimentary language, but whose arithmetical skills are still efficient. An even more striking case, whose calculating skills are at the near-prodigy level, has been recently reported by Remond-Besuchet, Noël, Seron, Thioux, Brun, and Aspe (1999). The damage in patients with severe numerical difficulties involves the left parietal lobe, especially the inferior lobule: not an area thought to be implicated in core language abilities.

On the other hand, it could be argued that the relative independence of numerical and linguistic abilities in the adult brain may not reflect dependence on language in development. Brain specialization may emerge as the child acquires more language and more numerical knowledge. However, it has now been established in several different experimental paradigms that the prelinguistic infant is sensitive to the numerosity of visual displays (Antell \& Keating, 1983; Starkey \& Cooper, 1980; Van Loosbroek \& Smitsman, 1990), and from other studies, it seems plausible that infants can compare two num- 
erosities (Wynn, 1992). These findings suggest that brain specialization for the discrimination of numerosities, at least, has occurred by birth. To assess the plausibility of genetically encoded specialization for numbers, we have studied the basic linguistic and numerical abilities in subjects with Turner's syndrome, a genetic abnormality known to affect mathematical performance (Rovet, Szekely, \& Hockenberry, 1994).

A population of nine X-monosomic females from 18 to 28 years of age was studied. All subjects were relatively high functioning with full-scale IQs in the normal or superior range. Brain morphology was examined using MRI voxel-based morphometry.

With regard to language abilities, all subjects performed in the normal or superior range on tests of naming, word, and sentence comprehension; of reading; and of spelling. None reported any early abnormality on language tasks.

Numerical abilities were evaluated in two ways.

(1) E (education-based) tasks: Performance on tasks that would seem to depend heavily on education and practice, such as single and multidigit arithmetic, both speeded oral and written.

(2) B (biologically based) tasks: Performance on tasks that may reflect basic, inherited, numerical abilities, in particular visual numerosity judgments (subitizing) and number magnitude comparisons.

All subjects showed abnormality in numerical performance on E tasks. In particular, they took at least twice as long as controls on simple fact retrieval tasks. It has been argued that these facts, such as those in multiplication tables, are stored in a verbal format (Dehaene \& Cohen, 1995). Since no linguistic deficit was discernible, this raises a problem for that theory.

On B tasks, some but not all of the subjects showed deficits.

Voxel-based morphometry showed no area of reduced gray matter common to all subjects. However, there were systematic differences according to whether the $\mathrm{X}$ chromosome was inherited from the mother $(\mathrm{Xm})$ or the father $(\mathrm{Xp})$. The $\mathrm{Xm}$ karyotype all showed reduced gray matter relative to similar age female controls in the left precuneus/intraparietal sulcus, while the Xp karyotype showed reduced gray matter in SMA, anterior cingulate, and left cerebellum.

\section{REFERENCES}

Antell, S. E., \& Keating, D. P. 1983. Perception of numerical invariance in neonates. Child Development, 54, 695-701.

Cipolotti, L., Butterworth, B., \& Denes, G. 1991. A specific deficit for numbers in a case of dense acalculia. Brain, 114, 2619-2637.

Dehaene, S., \& Cohen, L. 1995. Towards an anatomical and functional model of number processing. Mathematical Cognition, 1, 83-120.

Remond-Besuchet, C., Noël, M.-P., Seron, X., Thioux, M., Brun, M., \& Aspe, X. 1999. Selective preservation of exceptional arithmetical knowledge in a demented patient. Mathematical Cognition, 5. 
Rossor, M. N., Warrington, E. K., \& Cipolotti, L. 1995. The isolation of calculation skills. Journal of Neurology, 242, 78-81.

Rovet, J., Szekely, C., \& Hockenberry, M.-N. 1994. Specific arithmetic calculation deficits in children with Turner syndrome. Journal of Clinical and Experimental Neuropsychology, 16, 820-839.

Skuse, D. H., James, R. S., Bishop, D. V. M., Coppin, B., Dalton, P., Aamodt-Leeper, G., Bacarese-Hamilton, M., Creswell, C., McGurk, R., \& Jacobs, P. A. 1997. Evidence from Turner's syndrome of an imprinted X-linked locus affecting cognitive function. Nature, 387, 705-708.

Starkey, P., \& Cooper, R. G., Jr. 1980. Perception of numbers by human infants. Science, 210, 1033-1035.

Van Loosbroek, E., \& Smitsman, A. W. 1990. Visual perception of numerosity in infancy. Developmental Psychology, 26, 916-922.

Wynn, K. 1992. Addition and subtraction by human infants. Nature, 358, 749-751.

\section{Functional Neuroanatomy of the Semantic System: The Case for Numerals}

\section{Marc Thioux, Xavier Seron, and Mauro Pesenti \\ Unité de Neuropsychologie Cognitive, Université de Louvain, Belgium}

Recent functional neuroimaging studies suggest that specific brain regions may be differentially involved in processing certain semantic categories such as living things (e.g., animals) or artifacts (e.g., tools). In this study, we used PET to test the hypothesis of a segregation between the brain regions involved in the semantic processing of numerals and those involved in the semantic processing of another category of words (animals). This hypothesis takes advantage of a double dissociation observed in neuropsychology. On one hand, Thioux et al. (1998) have described a patient who retained preserved ability to understand and produce numerals in various tasks despite a clear semantic deficit and a very severe anomia for other categories of words. On the other hand, Cipolotti et al. (1991) described the reverse dissociation, that is, a patient who was unable to understand and produce numerals whatever the task although she retained preserved semantic abilities for other categories of words. On the same line, at the anatomical level, many results in the literature point toward the left temporal lobe (mainly the medial and inferior temporal gyri) as being implicated in the semantic processing of words, whereas numerical processing has been traditionally located in the parietal lobe. Nonetheless, the available evidence for the parietal lobe involvement in numerical processing is still under debate. First, in most neuroimaging studies investigating numerical processing, there was no proper control of nonspecific parietal activation linked to the involvement of verbal short-term memory or some visuospatial processing. Second, in one of the 
most carefully designed PET studies, Dehaene et al. (1996) did not get the expected parietal activation during a magnitude comparison task, which is supposed to require the activation of the semantic representation of numerals. Our study contributes to this debate by evaluating and contrasting the involvement of the parietal lobe in magnitude comparison and parity judgment. Furthermore, we provide a direct comparison between the processing of numerals and the processing of words from the category of living things. To our knowledge, this comparison has not been investigated using PET. The tasks used to contrast the semantic of animals and that of numerals were chosen to be as similar as possible. We opposed the magnitude comparison on numerals with a comparison of ferocity for animals and the parity judgment on numerals with a judgment on the category of animals (mammal or not). The use of two different kinds of semantic tasks in each category (a comparison task and a categorization task) allowed us to contrast the effect of varying the semantic domain with the effect of varying the task requirement within that domain.

Eight healthy male volunteers underwent 14 successive scans after injection of ${ }^{15} \mathrm{O}$-labeled water. During the first and the last scans, the subjects were asked to rest silently with eyes closed. During the other scans, they performed a visuoperceptive task (control condition) and two semantic tasks (classification task and comparison task) on two types of material (verbal numerals and animal names), resulting in six active conditions each repeated twice. In the visuoperceptive task, the subjects had to decide whether the letters of the stimulus appearing on the screen of a computer (verbal numeral or animal name) were "plain" or "outlined." In the semantic classification tasks the subjects had to classify verbal numerals as "even or not" or animal names as "mammals or not." In the comparison tasks, the subjects had to classify verbal numerals as "larger than 5 or not" or animal names as "more ferocious than a dog or not.' PET and T1-weighted MR images were acquired and spatially normalized. Preliminary analyses have been performed on six subjects using SPM96. Response locations were interpreted in accordance with Talairach and Tournoux's (1988) Co-planar Stereotaxic Atlas.

A cognitive conjunction revealed the areas more activated during the semantic processing of numerals than during the semantic processing of animals, in both semantic tasks. Significant activation foci were found bilaterally in the intraparietal sulci (BA7), the right temporal gyrus (BA37), and the upper part of the left precentral gyrus (BA4). Other conjunctions revealed the areas involved in the comparison and classification processes relative to the visuo-perceptive tasks, irrespective of the semantic domain. Comparison activated the right middle frontal gyrus (BA10/46), the left posterior cingulate gyrus (BA31/23), and the left inferior precentral gyrus (BA6/4), whereas classification activated the right anterior cingulate gyrus (BA32) and the left inferior and middle frontal gyri (BA46).

In conclusion, our study confirms the segregation of the brain areas in- 
volved in the semantic processing of numerals as compared to other semantic categories and suggests a central role of the intraparietal sulci in the semantic processing of numerals.

\title{
REFERENCES
}

Cipolotti, L., Butterworth, B., \& Denes, G. 1991. A specific deficit for numbers in a case of dense acalculia. Brain, 114, 2619-2637.

Dehaene, S., Tzourio, N., Frak, V., Raynaud, L., Cohen, L., Mehler, J., \& Mazoyer, B. 1996. Cerebral activations during number multiplication and comparison: A PET study. Neuropsychologia, 34, 1097-1106.

Talairach, J., \& Tournoux, P. 1988. Co-planar stereotaxic atlas of the human brain. New York: Thieme.

Thioux, M., Pillon, A., Samson, D., de Partz, M-P., Noël, M-P., \& Seron, X. 1998. The isolation of numerals at the semantic level. Neurocase, 4, 371-389.

\section{Mental Calculation and Number Word Repetition: A PET Study}

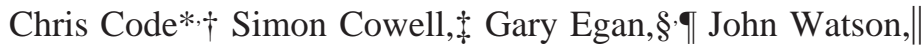 \\ Jenny Harasty**
}

*School of Communication Sciences \& Disorders, $\$$ School of Medical Radiation Science, and \|Neuropsychology Unit, Royal Prince Alfred Hospital, University of Sydney, Australia;

†School of Psychology, University of Exeter, United Kingdom; \$PET Centre, Austin

Repatriation Medical Centre, Melbourne, Australia; IHoward Florey Institute,

University of Melbourne, Australia; and **Prince of Wales, Medical Centre, University of New South Wales, Australia

Introduction. This PET study addressed the issue of mental calculation and attempted to separate linguistic (phonological) from nonlinguistic components.

Dehaene (1992) suggests that simple calculation (e.g., $6 \times 2$ ) first requires that the arithmetic facts are transformed into a phonological code to access the appropriate phonological fact stored in long-term memory (e.g., /six times two/), which is linked directly to routines for parsing auditory or written sequences of number words and to output routines for writing or speaking numbers. More complicated processes are likely to occur when calculation requires more than simple arithmetic facts retrieval (McCloskey et al., 1985). Mental calculation involves processing of the arithmetic symbol and retrieval of arithmetic facts, as well as the execution of arithmetic procedures.

Methods. Twelve right-handed subjects (mean age 48 years; range 27 to 67 years) including 6 males and 6 females participated. All subjects had more than 13 years of education.

A number repetition condition (A) and a calculation condition (B) were presented to subjects in a parametric design from prerecorded tape over ear- 
phones. There were 12 runs of 16 stimulus-response pairs randomly presented to each subject. Pairs entailed either oral repetition of auditorally presented number or the computation and oral report of a calculation presented auditorally.

The 12 runs contained different amounts of either repetition or calculation tasks randomly presented, but equal amounts of number repetition and calculation over the 12 runs. The amounts of either repetition or calculation tasks in a run ranged from 0 to 16 in increments of 2 .

Stimuli and responses were matched for syllabic length across conditions. Thus the repetition stimulus "say 18" and the calculation stimulus "six plus twelve" are identical in syllabic length, as is the response. Latencies between presentations, average acoustic intensity, and fundamental frequency were equal, so that when one condition was subtracted from the other what remained was what was not shared by the two conditions. Repetition trials varied in length between 2 and 8 syllables, such as "say 6" or "now can you please say 24." Calculation involved auditory comprehension, mental calculation, and generation and vocalization of the calculation result (all required responses were between 1 and 30).

Relative regional cerebral blood flow was measured by recording the distribution of cerebral radioactivity following intravenous injection of $\mathrm{H}_{2}{ }^{15} \mathrm{O}$, and a high-resolution magnetic resonance image (MRI) scan was also acquired for each subject. An average PET image for each subject was formed and was aligned to his or her segmented MRI image. Statistical parametric mapping (SPM95) was used to identify voxels which had significant correlation values with either the repetition or the calculation condition. Transformations to Talaraich space (Talaraich and Tourneaux, 1988) were calculated.

Results and discussion. For number repetition statistically significant brain activation was observed bilaterally in the primary and secondary auditory cortex and the primary motor mouth cortex, including the left and right superior temporal gyri, left and right precentral gyri, left lower frontal gyrus, left medial frontal gyrus (SMA), and anterior-superior left cerebellum.

During calculation main significant brain activations were bilaterally in the cingulate, the left frontal and left prefrontal cortex, the posterior left inferior medial and frontal gyri (BA 45), the left precentral gyrus, the right superior-anterior temporal, and the left inferior parietal (the supramarginal gyrus) gyri. Small focal activations were observed in the medial thalamus, the superior brain stem, and the right putamen.

Cingulate activity has been found in PET studies of calculation (Dehaene et al., 1996; Costello et al., 1997) and in our study is probably due to the significantly increased attentional demands. The left inferior parietal region, including the anterior supramarginal gyrus, has been identified in a range of studies as active during arithmetic processing and as a common lesion site resulting in acalculia.

Lesion and PET studies have produced converging data on the involve- 
ment of working memory in calculation supporting a left prefrontal (Broca's area) and left parietal (supramarginal gyrus) involvement in working memory responsible respectively for subvocal rehearsal (articulatory loop) and phonological storage (Burbaud et al., 1995; Paulescu et al., 1993; Perani et al., 1993).

During repetition subjects attended to continuous speech of between 2 and 8 syllables before repetition. Consequently, we anticipated significant activation of areas associated with auditory as well as motor processing.

In conclusion, mental calculation appears to engage a network in the left and right hemispheres underlying attention, auditory processing, and motor processing and engaging longer term phonological storage, activating the supramarginal gyrus where execution of the calculation occurs.

\section{REFERENCES}

Burbaud, P., Camus, O., Guehl, D., Muller, E., Bioulac, B., Caille, J. M., \& Allard, M. 1997. Influence of procedure and strategy on lateralization of cortical activation during number processing. NeuroImage Supplement, 5, 104.

Costello, P. A., Lee, J. T., \& Pardo, J. V. 1997. Retrieval of facts from arithmetic tables: A PET study. NeuroImage Supplement, 5, 105.

Dehaene, S. 1992. Varieties of numerical abilities. Cognition, 44, 1-42.

McCloskey, M. 1992. Cognitive mechanisms in numerical processing: Evidence from acquired dyscalculia. Cognition, 44, 107-157.

Paulesu, E., Frith, C. D., \& Frackowiak, R. S. J. 1993. The neural correlates of the verbal components of working memory. Nature, 362, 342-344.

Perani, D., Bressi, S., Cappa, S. F., Vallar, M., Grassi, F., Caltagirone, C. Cipolotti, L., Franceschi, M., Lenzi, G. L., \& Fazio, F. 1993. Evidence of multiple memory systems in the human brain. Brain, 116, 903-919.

Talairach, J., \& Tournoux, P. 1988. Co-planar stereotaxic atlas of the human brain. New York: Thieme.

\section{Language and Elementary Arithmetic: Dissociations between Operations}

\section{Stanislas Dehaene and Laurent Cohen}

Inserm U334, Service Hospitalier Frédéric Joliot, CEA/DRM/DSV, Orsay, and Service de Neurologie 1, Hôpital de la Salpêtrière, Paris, France

In many single-case studies of patients with number-processing deficits, different arithmetic operations such as naming, comparing, multiplying, and subtracting digits are not equally impaired. Most counterintuitively, even two seemingly similar arithmetic operations, such as subtraction and multiplication, can be dissociated. In this presentation, we briefly review the available evidence for such dissociations in brain-lesioned patients, and we pro- 
pose a theoretical hypothesis about their origins. We then describe brainimaging experiments in normal subjects which confirm that distinct cerebral circuits are recruited for different arithmetic operations. The results clarify the cerebral substrates of number processing.

Our theoretical account of dissociations between operations derives from the triple code of number processing. We postulate that familiar multiplication problems are often learned by rote and solved through the retrieval of automatic verbal associations. Other problems, particularly subtraction problems, are not systematically memorized and require the manipulation of the quantities represented by the operands. Thus, these two types of arithmetic routines (verbal vs semantic) can be doubly dissociated in brain-damaged patients with acalculia.

A disproportionate impairment of multiplication compared to subtraction may be taken as an index of the verbal origin of anarithmetia and can result from different functional mechanisms, corresponding to distinct lesion sites. (1) In pure alexia following left inferotemporal lesions, Arabic stimuli cannot be translated into words, a prerequisite for retrieving stored multiplication facts. For instance, patient VOL read the problem $5 \times 9$ as " $4 \times 6$ ", and therefore retrieved the erroneous result ' 24 .' ' In contrast, she could solve subtraction problems by accessing and manipulating the quantities represented by Arabic operands, without a prior encoding in verbal format. For instance, when presented visually with the subtraction problem $8-6$, patient VOL read the problem aloud as " $5-4$,' but nevertheless produced the correct result " 2 .', (2) Lesions affecting the language cortex can impede the ability to represent multiplication problems as word strings and hence preclude the retrieval of the appropriate results. (3) Left subcortical lesions can interfere with the general ability to retrieve stored verbal automatisms, including familiar multiplication facts.

The opposite dissociation between operation types, i.e., preserved multiplication with severely impaired subtraction, results from deficits affecting the semantic or quantitative component of elementary arithmetic processing. In such cases, anarithmetia is part of a more general impairment of quantity manipulation, visible for instance in number comparison or bisection tasks. It is essential to note that this type of "semantic anarithmetia" can occur in the absence of any impairment of reading or writing or more generally of any deficit in the verbal domain. It is often associated with other features of Gerstmann's syndrome and results from lesions classically affecting the inferior parietal lobule, possibly the cortex surrounding the intraparietal sulcus.

Recently, we have used fMRI to examine the active cerebral networks while subjects perform various numerical tasks. In a first experiment, we contrasted single-digit naming, comparison, multiplication, and subtraction with a control task of letter naming. A distributed network of brain regions including parietal, frontal, and anterior cingulate areas was engaged during 
number processing. However, there were important differences as a function of task demands. First, the parietofrontocingular network was activated only when subjects were engaged in active number manipulations tasks (comparison, multiplication, or subtraction) and not in simple digit naming relative to the letter naming control. Second, although the circuit was already engaged bilaterally during the number comparison task relative to control, the four numerical tasks could be ordered hierarchically in the order Naming $<$ Comparison $<$ Multiplication $<$ Subtraction, so that each higher level task added a specific activation to the immediately lower task. Relative to digit naming, comparison activated only the depth of the right postcentral sulcus. Relative to comparison, multiplication caused a strong additional left intraparietal activation. Finally, relative to multiplication, subtraction yielded greater right postcentral and bilateral prefrontal activation. These results establish both the existence of a parietofrontocingulate network active during various mental arithmetic tasks and its variable involvement as a function of task demands.

In a second experiment, we directly tested the hypothesis that the parietal regions are involved in quantity processing and are not necessary for rote retrieval of exact arithmetic facts. Subjects performed superficially similar addition tasks, but one task required retrieving an exact result, while the other required approximating the result. On each trial, an addition problem and then two candidate answers were flashed. Subject selected either the correct answer (exact task) or the most plausible answer (approximate task) by depressing the corresponding hand-held button as quickly as possible. The results indicated that the bilateral parietofrontocingular network is significantly more active during approximation than during exact calculation. Conversely a left lateralized network, implicating in particular the left inferior frontal region, was more active during exact calculation than during approximation.

In conclusion, our results indicate that even within the small domain of elementary arithmetic, multiple mental representations are used for different tasks. The representation used for storing rote knowledge of exact arithmetic facts is language-specific and relies in part on left-lateralized perisylvian language circuits. In contrast, arithmetic operations that require quantity manipulations, such as subtraction or approximation rely primarily on visuospatial networks of the left and right parietal lobes and shows little or no dependence on language.

\section{REFERENCE}

Dehaene, S., \& Cohen, L. 1995. Towards an anatomical and functional model of number processing. Mathematical Cognition, 1, 83-120. 